\title{
GPS Pull-In Search Using Reverse Directional Finite Rate of Innovation (FRI)
}

\author{
Seung-Hyun Kong, Kyungwoo Yoo
}

The CCS Graduate School for Green Transportation in the Korea Advanced Institute of Science and Technology (KAIST), Daejeon 305-701, Korea

\begin{abstract}
When an incoming Global Positioning System (GPS) signal is acquired, pull-in search performs a finer search of the Doppler frequency of the incoming signal so that phase lock loop can be quickly stabilized and the receiver can produce an accurate pseudo-range measurement. However, increasing the accuracy of the Doppler frequency estimation often involves a higher computational cost for weaker GPS signals, which delays the position fix. In this paper, we show that the Doppler frequency detectable by a long coherent auto-correlation can be accurately estimated using a complex-weighted sum of consecutive short coherent auto-correlation outputs with a different Doppler frequency hypothesis, and by exploiting this we propose a noise resistant, low-cost and highly accurate Doppler frequency and phase estimation technique based on a reverse directional application of the finite rate of innovation (FRI) technique. We provide a performance and computational complexity analysis to show the feasibility of the proposed technique and compare the performance to conventional techniques using numerous Monte Carlo simulations.
\end{abstract}

Keywords: pull-in state, finite rate of innovation

\section{INTRODUCTION}

Successful positioning in harsh Global Positioning System (GPS) environments has been one of the most challenging problems in GPS, and the demand for location-based service (LBS) in harsh environments for GPS, such as dense urban and indoor environments is continuously increasing. To meet the needs for GPS in harsh environments, GPS receivers may need a faster and higher sensitivity acquisition and tracking functions that can produce accurate carrier phase and code phase estimates in various GPS signal conditions. In the literature, a number of acquisition techniques for weak GPS signals have been introduced. For example, a GPS receiver connected to a synchronized cellular communication network (Kransner 1998, van Diggelen 2009) can make use of the downlink signal measurements and downlink information from the

Received July 09, 2014 Revised July 31, 2014 Accepted Aug 01, 2014

†Corresponding Author

E-mail: skong@kaist.ac.kr

Tel: +82-42-350-1265 Fax: +82-42-350-1250 cellular base station to quickly acquire GPS signals with high sensitivity. On the other hand, conventional techniques to enhance the acquisition sensitivity and to reduce the mean acquisition time (MAT) such as long coherent integration and non-coherent accumulation techniques (Parkinson et al. 1996) have been continuously studied, and, recently, techniques such as parallel signal search, FFT-based signal search (Borre et al. 2007), and two-dimensional compressed correlator (Kong \& Kim 2013) techniques have been developed.

To produce accurate measurements of code phase and carrier phase of incoming GPS signals, GPS receivers employ tracking functions that require initial code phase and Doppler frequency estimates with sufficient accuracy. In practice, the accuracy required for the initial resolutions of the code phase and Doppler frequency are less than a half chip and about ten Hertz (Tsui 2005), respectively. Since most of the acquisition techniques perform Doppler frequency search for incoming GPS signals with a search step size about $1 /(2 T) \mathrm{Hz}$, where the coherent integration time $T$ is usually $1 \sim 5 \mathrm{~ms}$, the Doppler frequency estimate may have an error of up to $250 \mathrm{~Hz}$, which can be too coarse for a carrier phase tracking loop. However, an initial code 
phase error of up to a half chip can be tolerable for most of the code phase tracking functions (Irsigler \& Eissfeller 2003). Therefore, a GPS receiver has a pull-in state between the acquisition and tracking states to increase the accuracy of the Doppler frequency estimate to a high enough level to accurately run carrier phase tracking. In the pull-in state, a GPS receiver tries to obtain a fine Doppler frequency, carrier phase, and $C / N_{0}$ estimates of an incoming GPS signal, which can take about 1.5 s (Kelley et al. 2002). To improve the pull-in search performance, conventional techniques, such as Tsui (2005) and Goiser \& Berger (1996), perform a fast Fourier transform (FFT) to find the maximum frequency component from code wiped-off sample data and then utilize the amplitude of the component to obtain a fine Doppler frequency estimate. Consecutive fine Doppler frequency estimates can be averaged to increase accuracy in the presence of noise, but, in general, the conventional techniques work well for high $C / N_{0}$. To lessen the noise effect on the Doppler frequency estimation, Sagiraju et al. (2006) suggest increasing $T$ up to $5 \mathrm{~ms}$, however, the scheme requires a smaller initial Doppler frequency estimation error than $50 \mathrm{~Hz}$, which, in effect increases the complexity of the acquisition state. On the other hand, Zeng \& Li (2010) suggest a serial Doppler frequency search using correlation in the time domain, and employ a curve fitting algorithm to find a more accurate Doppler frequency estimate. The curve fitting may be able to provide good Doppler frequency and carrier phase estimates at the cost of increased computational complexity. In general, since a GPS receiver does not yet have the knowledge on the received data bit in the pull-in state, increasing the accuracy of Doppler frequency and carrier phase estimates of weak GPS signals can be a complex problem due to the computational complexity and algorithmic complexity required to mitigate the effect of bit transition. In practice, the carrier phase estimation is performed by the phase locked loop in the tracking state.

Despite the complexity and importance, there has been little attention paid to the pull-in search of GPS receivers in the literature. To reduce the computational complexity and improve the accuracy of the Doppler frequency and carrier phase estimates in the pull-in state, we propose a reverse-directional finite rate of innovation (FRI) technique with iterative Cadzow denoising applied to consecutive coherent auto-correlation function (ACF) outputs to reduce the computational complexity in estimating the Doppler frequency and carrier phase of an incoming GPS signal with high accuracy and to increase noise robustness. The complexity of the proposed technique is analyzed and the performance of the proposed technique is compared to the conventional techniques using numerous Monte Carlo simulations.

The rest of this paper is organized as follows. In Section 2 , we introduce a general continuous form expression for a coherent ACF output, and in Section 3, we verify that a long coherent ACF output for a Doppler frequency can be estimated with consecutive short coherent ACF outputs at a close Doppler frequency. Section 4 provides the details and complexity of the proposed technique that exploits the findings in Section 3, and Section 5 shows the analysis of the performance of the proposed technique and a performance comparison to conventional techniques using numerous Monte Carlo simulations. And a conclusion is drawn in Section 6.

Throughout this paper, the following conventions are used for notation. Vectors or matrices are denoted by boldface symbols. Small letters are used for scalars and vectors, and capital letters are used for matrices.

\section{COHERENT ACF OUTPUT OF GPS SIGNAL}

Let $r(t)$ be the frequency down-converted incoming $L 1$ GPS coarse acquisition (C/A) code signal to an IF frequency $f_{I F}$, then the complex IF signal can be expressed as

$$
r(t)=\sum_{q=1}^{Q} A_{q} D_{q}\left(t-\tau_{q}\right) C_{q}\left(t-\tau_{q}\right) e^{j\left[2 \pi\left(f_{I F}+f_{D}^{q}\right) t+\phi_{q}\right]}+n(t),
$$

where $\mathrm{Q}$ is the number of GPS satellite signals being received, $A_{q}, \tau_{q},\left|f_{D}^{q}\right| \leq(5 \mathrm{kHz})$, and $\phi_{q}$ are the slowly varying amplitude, code phase, Doppler frequency, and unknown carrier phase of the q-th satellite signal, respectively, $D_{q}(t)$ and $C_{q}(t)$ are the navigation data bit signal and PseudoRandom Noise code signal of the q-th satellite at time $t$, respectively, and $\mathrm{n}(\mathrm{t})$ represents an additive white Gaussian noise with two-sided power spectral density (PSD) $N_{0} / 2$. Note that the data bit and chip rates are $R_{b}(=50 \mathrm{bps})$ and $R_{c}$ (= $1.023 \mathrm{Mcps}$ ), respectively, and that the $\mathrm{C} / \mathrm{A}$ code has code length $L_{c}=1023$ chips so that the code period is only $T_{1}(=1$ $\mathrm{ms})$. When the coherent integration (correlation) length used in the acquisition state is $T=L_{T} T_{1}$, where $L_{T}$ is an integer much larger than 1 , and code phase search resolution is $T_{c} / 2$, the detected code phase $\tau_{q}$ and Doppler frequency $f_{D}^{q}$ at the acquisition state can have errors upto $T_{c} / 4$ and $1 /$ $(4 T)$, respectively. Note that the navigation data remains unknown in the pull-in state. To detect and track the code phase $\tau_{q}$ and Doppler frequency $f_{D}^{q}$ of the q-th satellite signal using correlation, the receiver generates a prompt replica signal 


$$
r_{q}(t)=C\left(t-\hat{\tau}_{q}\right) e^{j 2 \pi\left(f_{I F}+\hat{f}_{D}^{q}\right) t}
$$

where $\hat{\tau}_{q}$ and $\hat{f}_{D}^{q}$ are the code phase and carrier frequency estimates made by the receiver's acquisition function, respectively. The receiver integrates the product of $r(t)$ and $r_{q}^{*}$ $(t)$ (complex conjugate of $r_{q}(t)$ ) over the coherent integration interval $T$ to see the correlation between the two functions. Defining $\delta \tau=\tau_{q}-\hat{\tau}_{q}$ and $\delta f=f_{D}^{q}-\hat{f}_{D}^{q}$, the mathematical expression of the ACF output over the two-dimensional space is readily available from Kong \& Kim (2013).

$$
\begin{aligned}
R_{2}(\delta \tau, \delta f)= & \frac{1}{T} \int_{t_{0}}^{t_{0}+T} r(t) r_{q}^{*}(t) d t \\
= & \frac{A_{q} D_{q}}{T} \int_{t_{0}}^{t_{0}+T} C\left(t-\tau_{q}\right) C\left(t-\hat{\tau}_{q}\right) e^{j(2 \pi \delta f t+\phi)} d t+w \\
= & \frac{A_{q} D_{q} \sin (\pi \delta f T)}{\pi \delta f T} \times \frac{\sin \left(\pi \delta f\left(T_{c} \dashv \delta \tau \mid\right)\right)}{\sin \left(\pi \delta f T_{c}\right)} \\
& \times e^{j\left(2 \pi \delta f t_{0}+\pi \delta f T-\pi \delta f|\delta \tau|+\phi\right)}+w
\end{aligned}
$$

where $D_{q}\left(t-\tau_{q}\right)$ is assumed as constant $D_{q}$ for the integration interval $T$, and

$$
\begin{aligned}
w= & \frac{1}{T} \int_{t_{0}}^{t_{0}+T}\left[\sum_{p=1, p \neq q}^{Q} A_{p} D_{p}\left(t-\tau_{p}\right) C_{p}\left(t-\tau_{p}\right)\right. \\
& \left.\times e^{j\left[2 \pi\left(f_{I F}+f_{D}^{p}\right) t+\phi_{p}\right]}+n(t)\right] r_{q}(t)^{*} d t \\
; & \frac{1}{T} \int_{t_{0}}^{t_{0}+T_{1}} n(t) r_{q}(t)^{*} d t
\end{aligned}
$$

is a complex Gaussian noise with two-sided power spectral density $N_{0} / 2$, since signal powers are negligible when compared to the noise power. When $T=T_{b}\left(=1 / R_{b}\right)$ and when $\phi, \delta \tau$, and $\delta f$ are small enough, $R_{2}(\delta \tau, \delta f) \geq 0$ indicates that $D_{q}=+1$, and $R_{2}(\delta \tau, \delta f)<0$ indicates that $D_{q}=-1$. Note that there are several techniques to remove the effect of the navigation data signal $D_{q}(t)$ from $R_{2}(\delta \tau, \delta f)$; a number of bit sequences are estimated using Kalman filter (Psiaki \& Jung 2002), the binary value of $D_{q}(t)$ is estimated by testing different bit combinations (Petovello et al. 2008), or with the assistance information from the cellular base station in the AssistedGPS system (Kransner 1998, van Diggelen 2009). Since figuring out the data $D_{q}(t)$ is one of the primary tasks in the tracking state, we neglect the effect of the unknown data in this paper.
In this section, we break $R_{2}(\delta \tau, \delta f)$ obtained with a long coherent interval $T \gg T_{1}$ into $L_{T}\left(=T / T_{1}\right)$ consecutive $R_{2}(\delta \tau$, $\delta f$ )s obtained with short coherent interval $T_{1}$ and investigate the possibility of precisely estimating the Doppler frequency of $r(t)$ with the $L_{T} R_{2}(\delta \tau, \delta f)$ s obtained. In the following analysis, we drop the subscript $(\cdot)_{q}$ (unless necessary) for simplicity in algebraic expressions.

Let the input to the pull-in state be denoted by $R_{2}(\delta \tau$, $\delta f)=\left\{R_{2}^{1}(\delta \tau, \delta f) \mid 1=0,1, \ldots, L_{T^{-}}-1\right\}$, where

$$
\begin{aligned}
R_{2}^{l}(\delta \tau, \delta f)= & \frac{A}{T_{1}} \int_{l T_{1}}^{(l+1) T_{1}}\left[C(t-\tau) e^{j\left(2 \pi f_{D} t+\phi_{q}\right)}+n(t)\right] \\
& \times C(t-\hat{\tau}) e^{-j 2 \pi \hat{f}_{D}^{t}} d t \\
= & \frac{A \sin \left(\pi \delta f T_{1}\right) \sin \left(\pi \delta f\left(T_{c} \dashv \delta \tau \mid\right)\right)}{\pi \delta f T_{1}} \sin \left(\pi \delta f T_{c}\right) \\
& \times e^{j\left(\pi \delta f\left[2 t_{t}+T_{1}-\mid \delta \tau\right]+\phi\right)}+n_{1}^{l},
\end{aligned}
$$

is already available from Kong \& $\operatorname{Kim~(2013)~and~}(\cdot)^{l}$ represents that the coherent integration from $t=l T_{1}=t_{l}$ to $t=(l+1) T_{1}=t_{l}+T_{1}$, and

$$
n_{1}^{l}=\frac{1}{T_{1}} \int_{t_{l}}^{t_{l}+T_{1}} n(t) r_{q}^{*}(t) d t
$$

is a complex Gaussian process with two-sided PSD $N_{0} / 2$. Note that in Eq. (5) the expected ACF output for $|\delta \tau| \geq T_{c}$ is assumed as zero. When the code phase estimation is successful, i.e., $\delta \tau=T_{c} / 2$, the expression Eq. (5) can be simplified to a single-dimensional function

$$
\begin{aligned}
\left.R_{2}^{l}(\delta \tau, \delta f)\right|_{\delta \tau=0} & =\frac{A \sin \left(\pi \delta f T_{1}\right)}{\pi \delta f T_{1}} e^{j\left[\pi(2 l+1) \delta f T_{1}+\phi\right]}+n_{1}^{l} \\
& =R_{1}^{l}(\delta f)
\end{aligned}
$$

and let

$$
\begin{aligned}
R_{1}(\delta f) & =\sum_{l=0}^{L_{T}-1} \frac{R_{1}^{l}(\delta f)}{L_{T}} \\
& =\sum_{l=0}^{L_{T}-1}\left[\frac{A \sin \left(\pi \delta f T_{1}\right)}{\pi \delta f L_{T} T_{1}} e^{j\left[\pi(2 l+1) \delta f T_{1}+\phi\right]}+\frac{n_{1}^{l}}{L_{T}}\right] .
\end{aligned}
$$

when the carrier frequency of $r_{q}(t)$ is $\hat{f}_{D}+\Delta_{f}$, it is found from Eq. (7) that

$$
\begin{aligned}
R_{1}^{l}\left(\delta f-\Delta_{f}\right)= & \frac{A \sin \left[\pi\left(\delta f-\Delta_{f}\right) T_{1}\right]}{\pi\left(\delta f-\Delta_{f}\right) T_{1}} \\
& \times e^{j\left[(2 l+1) \pi\left(\delta f-\Delta_{f}\right) T_{1}+\phi\right]}+w^{l}\left(\Delta_{f}\right),
\end{aligned}
$$

where

$$
\begin{aligned}
w^{l}\left(\Delta_{f}\right) & =\int_{l T_{1}}^{(l+1) T_{1}} n(t) C(t-\hat{\tau}) e^{-j 2 \pi\left(\hat{f}_{D^{+}}{ }_{f}\right) t} d t \\
& ; e^{-j(2 l+1) \pi \Delta_{f} T_{1}} n_{1}^{l}
\end{aligned}
$$

\section{CONSECUTIVE SHORT COHERENT ACF OUTPUTS}


which is a complex Gaussian process with the same PSD to Eq. (6). Note that Eq. (10) holds only for a very small $\left|\Delta_{f}\right| T_{1}=1$. From Eqs. $(7,9,10)$, it is found that

$$
\begin{aligned}
R_{1}\left(\delta f-\Delta_{f}\right) & =\sum_{l=0}^{L_{T}-1} \frac{R_{1}^{l}\left(\delta f-\Delta_{f}\right)}{L_{T}} \\
& ; \frac{\beta\left(\delta f, \Delta_{f}\right)}{L_{T}} \sum_{l=0}^{L_{T}-1} R_{1}^{l}(\delta f) e^{-j(2 l+1) \pi \Delta_{f} T_{1}},
\end{aligned}
$$

which indicates that a long coherent ACF output $R_{1}\left(\delta f-\Delta_{f}\right)$ with a long coherent integration interval $T$ and a Doppler frequency estimate $\hat{f}_{D}+\Delta_{f}$ can be obtained from the linear sum of $L_{T}$ consecutive short coherent ACF outputs $R_{1}^{l}(\delta f)$ from $L_{T}$ integration segments $\left[l T_{1},(l+1) T_{1}\right]\left(l \in\left\{0,1, \ldots, R_{T}-1\right\}\right)$ of $\left[0, T_{1}\right]$ with a neighboring Doppler frequency $\hat{f}_{D}$, when $\left|\Delta_{f}\right|=1 / T_{1}$ and $\left|\delta f+\Delta_{f}\right|=1 / T_{1}$ are satisfied. In other words, Eq. (11b) states that it is possible to precisely estimate a long coherent ACF output $R_{1}\left(\delta f-\Delta_{f}\right)$ for a Doppler frequency hypothesis using consecutive short coherent ACF outputs $R_{1}^{l}(\delta f)\left(l \in\left\{0,1, \ldots, R_{T}-1\right\}\right)$ with a close Doppler frequency hypothesis. This observation is exploited in the proposed technique for an accurate and fast GPS pull-in search. In addition, it should be noted that the carrier frequency $\delta f$ of $\mathbf{R}_{1}(\delta f)\left(=\left[R_{1}^{0}(\delta f), R_{1}^{1}(\delta f), \ldots, R_{1}^{L_{T}-1}(\delta f)\right]\right)$ can be estimated by compensating the frequency $\delta f$ with $\Delta_{f}$ which maximizes

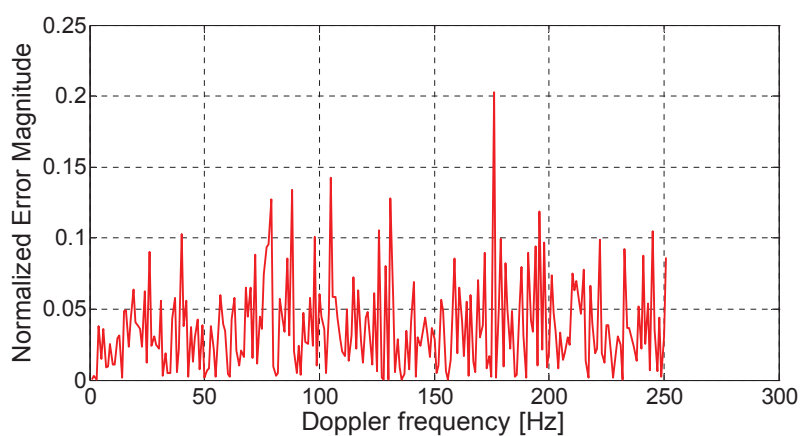

(a) Standard deviation of the normalized amplitude difference

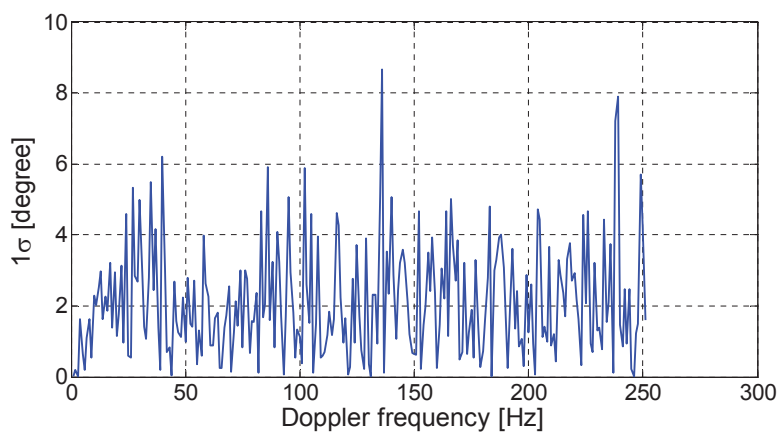

(b) Standard deviation of the phase difference

Fig. 1. Validity of the approximation in Eq. (11b). the linear sum in Eq. (11b).

Fig. 1 shows $10^{4}$ Monte Carlo simulation results demonstrating the estimation accuracy of the expression Eq. (11b) for a random Doppler frequency $\delta f$ of the incoming signal from $0 \mathrm{~Hz}$ to $250 \mathrm{~Hz}$, when $C / N_{0}=40 \mathrm{~dB}$ $\mathrm{Hz}$ and $L_{T}=2^{5}$. Fig. 1a shows that the result of absolute normalized amplitude difference, i.e., $\mid\left[R_{1}(0)-L_{T}^{-1} \sum_{l=0}^{L_{T}-1} R_{1}^{l}\right.$ $\left.(\delta f), \ldots, R_{1}^{L_{T}-1}(\delta f) \mathrm{e}^{-j(2 l+1) \pi \delta f T_{1}}\right] / R_{1}(0) \mid$ for $\delta f$ varying from 0 to $250 \mathrm{~Hz}$. Fig. $1 \mathrm{~b}$ shows that the standard deviation of the Doppler frequency difference between the true Doppler frequency and the estimated Doppler frequency using Eq. (11b) is small, also. As a result, it is demonstrated that $\mathbf{R}_{1}(\delta f)=\left\{R_{1}^{l}(\delta f) \mid l=0,1, \ldots, L_{T}-1\right\}$ is a set of samples of a noisy complex carrier signal, with a carrier frequency $\delta f$, amplitude $1 / L_{T}$, and phase $\pi \delta f T_{1}$, sampled at $1 / T_{1}[\mathrm{~Hz}]$, and that estimating the carrier frequency and phase of $\mathbf{R}_{1}(\delta f)$ can provide a mismatch of Doppler frequency and phase estimates between those of the incoming signal and the receiver replica. In addition, when the phase of $R_{1}^{l}(\delta f)$ is denoted by $\phi$ as in Eq. (7), the accumulation of $R_{1}\left(\delta f-\Delta_{f}\right)$ for all $\left(l \in\left\{0,1, \ldots, R_{T}-1\right\}\right)$ in Eq. (11a), i.e., the summation of $L_{T}$ consecutive short coherent ACF outputs, will have an overall phase $\phi-\pi \Delta_{f} T_{1}$. Therefore, when the frequency error is estimated, the phase of the incoming signal can be readily available from

$$
\phi=\angle R_{1}(0)=\angle R_{1}\left(-\Delta_{f}\right)+\pi \Delta_{f} T_{1} .
$$

consequently, we can see that estimating the frequency error $\delta f$ is to search for a solution $\Delta_{f}$ that maximizes $\mid R_{1}(\delta f$ $\left.\Delta_{f}\right) \mid$ such that

$$
\hat{\Delta}_{f}=\underset{\Delta_{f}}{\arg \max }\left|R_{1}\left(\delta f-\Delta_{f}\right)\right|
$$

which is equivalent to the frequency estimation of carrier signal samples $\mathbf{R}_{1}(\delta f)=\left\{R_{1}^{l}(\delta f) \mid l=0,1, \ldots, L_{T}-1\right\}$.

\section{FREQUENCY AND PHASE ESTIMATION USING THE PROPOSED TECHNIQUE}

There are a number of techniques that can provide a solution for the problem in Eq. (13). For example, instead of searching for $\Delta_{f}$, trivial techniques can be the Discrete Fourier Transform (DFT) and the FFT that can estimate the frequency of $\mathbf{R}_{1}(\delta f)$ by finding the maximum frequency component such that

$$
\tilde{\delta} f=\underset{\delta f}{\arg \max }\left|F F T\left\{R_{1}(\delta f)\right\}\right|,
$$


which may require only $L_{T} \log _{2} L_{T}$ complex multiplications. The estimate $\tilde{\delta} f$ is asymptotically unbiased for a very large $L_{T}$ (Hayes 1996), and the maximum error of the FFTbased scheme Eq. (14) is $1 /(2 T)[\mathrm{Hz}$. Since the frequency estimation error with the FFT (or DFT) is uniformly distributed in $[-1 /(2 T), 1 /(2 T)][\mathrm{Hz}]$, the absolute mean frequency estimation error $1 /(4 T)[\mathrm{Hz}]$ is not sufficiently accurate when $T$ is not very large. In this section, however, we propose a reverse directional FRI technique to increase the frequency estimation accuracy when $T$ is not very large.

\subsection{Proposed Reverse Directional FRI Technique}

In fact, the observation $\mathbf{R}_{1}(\delta f)$ is the vector of samples of a signal with FRI (Vetterli et al. 2002), i.e., the samples contain only a finite number $K(<\infty)$ of innovation of a periodic signal. For an example, a low pass filtered input signal $y(t)$ composed of $T_{P}$-periodic distinctive Dirac delta functions with complex weights $x_{k}(k=0,1, \ldots, k-1)$ as shown in Fig. 2a, the FRI technique (Vetterli et al. 2002), is to estimate the delays $t_{k}(k=0,1, \ldots, k-1)$ of the Dirac delta functions in the input using the minimum number of samples of $y(t)$. The estimation of $t_{k}(k=0,1, \ldots, k-1)$ is performed in the frequency domain, since the time domain samples of $y(t)$ are effectively the samples of the linear sum of complex sinusoids. The delays can be accurately estimated from the zeros of the annihilating filter $H[\mathrm{z}]$ that annihilates the samples of the linear sum of complex sinusoids. And the amplitude $x_{k}$ is estimated by solving the Vandermonde system of linear equations (Vetterli et al. 2002). On the contrary, the problem in Eq. (13) is to estimate the frequency of a single $(K=1)$
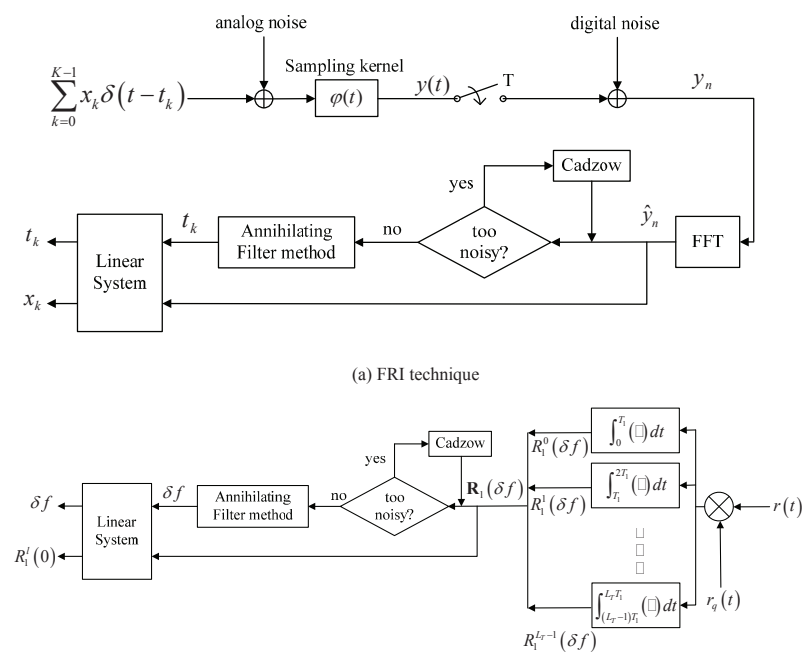

(b) Proposed FRI-based technique

Fig. 2. Reverse directional application of FRI for GPS. complex sinusoid that is equivalently a single Dirac delta function in the frequency domain, which means that the problem in Eq. (13) requires reverse directional application of the FRI technique as shown in Fig. $2 b$ and as investigated in Kim et al. (2011), for synthetic aperture radar (SAR) signal processing. In other words, $\mathbf{R}_{1}(\delta f)$ can be regarded as a set of noisy time domain samples of a single (i.e., $K=1$ ) Dirac delta function in the frequency domain as

$$
R_{1}^{l}(\delta f)=\left(\frac{A_{q} \sin \left(\pi \delta f T_{1}\right)}{\pi \delta f T_{1}} e^{j\left[\pi \delta f T_{1}+\phi\right]}\right) e^{j 2 \pi \delta f T_{1}}+n_{1}^{l},
$$

where the sampling is performed at every $t=l T_{1}$. Therefore, we can expect that the single Dirac delta function in the frequency domain is $T_{1}^{-1}$-periodic, and that the location and amplitude of the corresponding delta function in the frequency domain can indicate the carrier frequency and phase of the $\mathbf{R}_{1}(\delta f)$, respectively.

Exploiting Blu et al. (2008) for the case of noisy sample input, $\mathbf{R}_{1}(\delta f)$, when the input is arranged into a Toeplitz matrix

$$
\mathbf{A}=\left[\begin{array}{cccc}
R_{1}^{L}(\delta f), & R_{1}^{L-1}(\delta f), & \ldots, & R_{1}^{0}(\delta f) \\
R_{1}^{L+1}(\delta f), & R_{1}^{L}(\delta f), & \ldots, & R_{1}^{1}(\delta f) \\
\vdots & \vdots & \ddots & \vdots \\
R_{1}^{L_{T}-1}(\delta f), & R_{1}^{L_{T}-2}(\delta f), & \ldots, & R_{1}^{L_{T}-L-1}(\delta f)
\end{array}\right]
$$

where $L \geq K$, an annihilating filter

$$
H(z)=\sum_{k=0}^{1} h_{k} z^{-k}=1-e^{-j 2 \pi f_{1} T_{1}} z^{-1}
$$

that minimizes the total least squares

$$
\min _{H}\|H\|^{2}
$$

under a constraint $\|H\|^{2}=1$ provides the frequency estimate $\hat{\delta} f$, i.e., $f_{1}=\hat{\delta} f$. The coefficients of the annihilating filter $\left[h_{0}, h_{1}, \ldots, h_{k}\right]$ can be found from the eigenvector of $\mathbf{A}^{T} \mathbf{A}$ corresponding to the smallest eigenvalue, which is related to Pisarenko's method (Pisarenko 1973, Tufts \& Kumaresan 1982).

To increase the accuracy of the frequency estimate $\hat{\delta} f$ in the presence of heavy noise in the signal sample input, there can be two ways to increase the noise robustness of the algorithm in Eq. (18). One way is to increase $L_{T}$, which is a trivial solution, and the other way is to apply the iterative Cadzow denoising technique used in Blu et al. (2008). The first step of the technique is to perform a singular value decomposition (SVD) of A such that 


$$
\mathbf{A}=\mathbf{U S V}^{T},
$$

where $\mathbf{U}, \mathbf{S}$, and $\mathbf{V}$ are unitary, diagonal with elements in descending order, and unitary matrices of size $\left[\left(L_{T}-L\right) \times(L+1)\right]$, $[(L+1) \times(L+1)]$, and $[(L+1) \times(L+1)]$, respectively. When the $(K+1)$-th largest diagonal element of $\mathbf{S}$ is not sufficiently smaller than the $K$-th largest diagonal element, Cadzow's iterative denoising technique sets the $L-K+1$ smallest diagonal elements of $\mathbf{S}$ to zero to build a new diagonal matrix $\mathbf{S}^{(1)}$ in the first step, where $(\cdot)^{(i)}$ denotes the $i$-th iteration of the Cadzow denoising technique. In the second step, the technique constructs $\mathbf{A}^{(1)}$ using $\mathbf{S}^{(1)}$ as

$$
\mathbf{A}^{(1)}=\mathbf{U} \mathbf{S}^{(1)} \mathbf{V}^{T}
$$

The resulting $\mathrm{A}^{(1)}$ is not Toeplitz anymore, so the technique obtains the best Toeplitz approximation of $A^{(1)}$ by averaging the diagonals of $\mathbf{A}^{(1)}$ in the third step. The above three steps are usually iterated $N_{i}(>1)$ times until the $(K+1)$-th largest diagonal element of $\mathbf{S}^{\left(N_{i}\right)}$ becomes $R_{T H}(>>1)$ times smaller than the $K$-th largest diagonal element. From experimental observation, $R_{T H} \geq 50$ is sufficiently high. When it is found that the Cadzow denoising is completed after $N_{i}$-th iteration, $\mathbf{A}^{\left(N_{i}\right)}$ can be re-arranged to form a matrix $\mathbf{B}$ of size $\left[L_{T} \times 2\right]$ as

$$
\mathbf{B}=\left[\begin{array}{llll}
R_{1}^{1}(\delta f)^{\prime}, & R_{1}^{2}(\delta f)^{\prime}, & \ldots, & R_{1}^{L_{T}-1}(\delta f)^{\prime} \\
R_{1}^{0}(\delta f)^{\prime}, & R_{1}^{1}(\delta f)^{\prime}, & \ldots, & R_{1}^{L_{T^{-2}}}(\delta f)^{\prime}
\end{array}\right]^{T},
$$

where $R_{1}^{l}(\delta f)^{\prime}$ is the denoised $R_{1}^{l}(\delta f)$ by the iterative Cadzow denoising technique, and the eigenvector of $\mathbf{B}^{T} \mathbf{B}$ corresponding to the smallest eigenvalue provides the coefficients of the annihilating filter $\left[h_{0}, h_{1}\right]$. From Eq. (17) and for $K=1$,

$$
\hat{\delta} f=\left.f_{k}\right|_{k=1}=-\frac{1}{2 \pi T_{1}} \angle\left(\frac{-h_{1}}{h_{0}}\right)
$$

is the final estimate of the frequency $\delta f$ buried in $\mathbf{R}_{1}(\delta f)$ found by the proposed reverse directional FRI technique.

Once the frequency $\delta f$ is estimated, from Eq. (12), the relative phase of the input signal to the receiver generated carrier signal can be easily obtained as

$$
\hat{\phi}=\angle\left(\sum_{l=0}^{L_{T}-1} R_{1}^{l}(\delta f) e^{-j(2 l+1) \pi \hat{\delta} f T_{1}}\right)
$$

which is the arc-tangent of the frequency compensated and averaged In-phase and Quadrature-phase consecutive short coherent ACF outputs. Note that the proposed technique can estimate both the frequency and phase difference between the incoming signal $r(t)$ and the receiver replica signal, and that when there is no frequency, i.e., $\delta f=0$, the phase estimate of the proposed technique is similar to that of the conventional phase estimation technique.

\subsection{Realization and Receiver Complexity}

A drawback of the proposed technique for the pull-in search maybe the high computational cost for multiple SVD's in the iterative Cadzow denoising. To reduce the computational cost, computationally efficient SVD algorithms such as Riemannian SVD (R-SVD) and GolubReinsch SVD (GR-SVD), introduced in Chan (1982) can be applied as a solution. For an SVD of the matrix A of size $\left[\left(L_{T}-L\right) \times(L+1)\right]$, R-SVD and GR-SVD require

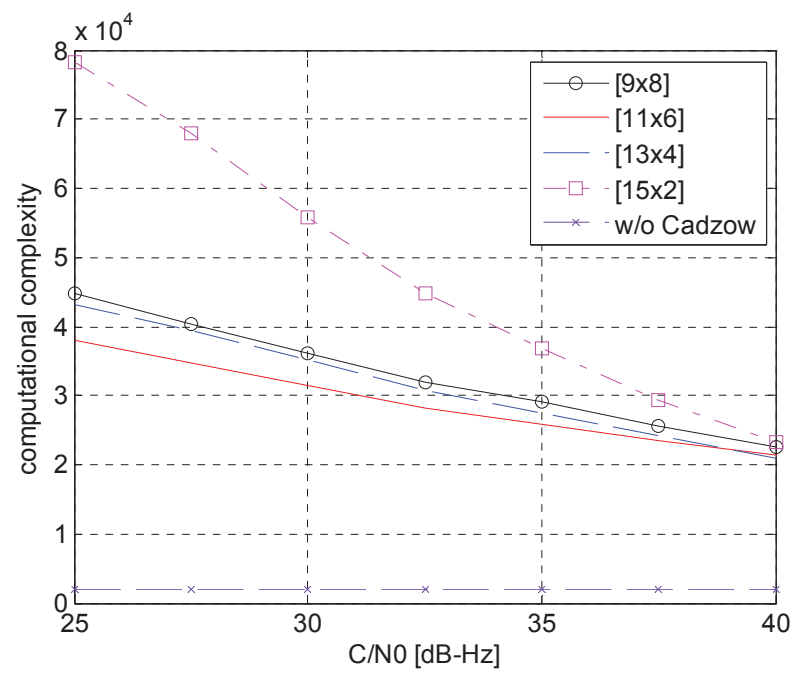

(a) $L_{T}=16$

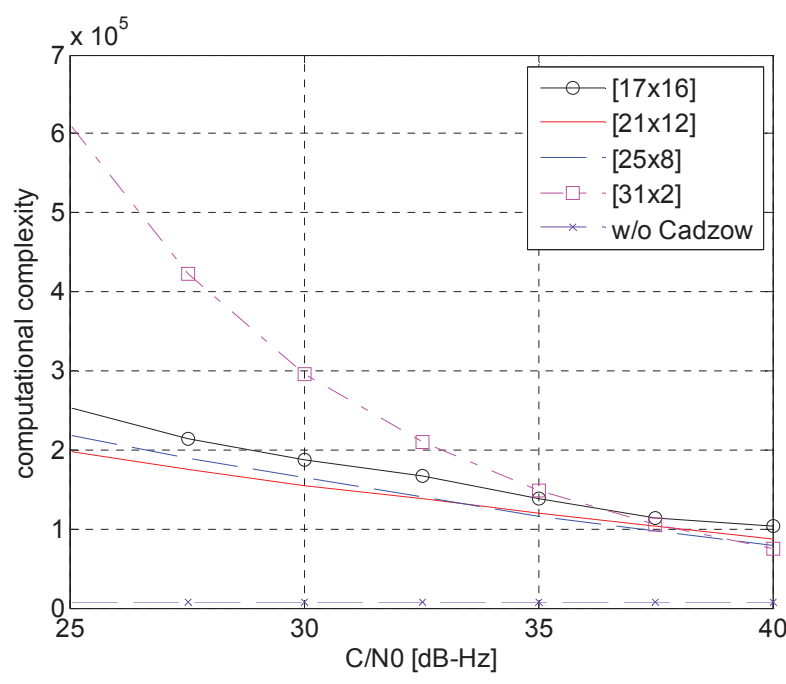

(b) $L_{T}=32$

Fig. 3. Complexity comparison. 


$$
\begin{aligned}
M_{1}= & {\left[4\left(L_{T}-L\right)^{2}+22(L+1)^{2}\right](L+1) } \\
& \text { for } L \text { sufficiently smaller than } L_{T} / 2, \\
M_{2}= & {\left[4\left(L_{T}-L\right)^{2}+8\left(L_{T}-L\right)(L+1)+9(L+1)^{2}\right](L+1) } \\
& \text { for } L \approx L_{T} / 2,
\end{aligned}
$$

respectively (Golub \& van Loan 1996). Assuming $L=L_{T} / 4$ for Eq. (24a) and $L=L_{T} / 2$ for Eq. (24b), and $L \gg>1$ for both cases, we can find that $M_{1} \approx M_{2} / 3$. Therefore, $1<L<<L_{T} / 2$ is a preferable choice, and the complexity decreases as $L$ decreases in R-SVD. Letting $1 / L_{T}<<\alpha_{l}<1 / 2$ and $L=\alpha_{l} L_{T^{\prime}}$ and assuming $N_{i}$ iterations of Cadzow denoising, the total computational complexity for the iterative Cadzow denoising is about

$$
M_{C D} \approx N_{i}\left(18 \alpha_{l}^{2}-8 \alpha_{l}+4\right) \alpha_{l} L_{T}^{3}
$$

where $N_{i} \leq 5$ is usually observed in experimentations (Blu et al. 2008). Notice that $M_{C D}$ in Eq. (25) is a monotonously increasing function with respect to $\alpha$, so that smaller $\alpha$ results in less computational cost. Fig. 3 shows the result of $10^{4}$ Monte Carlo simulations for a fixed $R_{T H}=50$ and for various $\alpha$ to estimate the total number of multiplications $M_{C D}$ in the proposed technique employing iterative Cadzow denoising with respect to GPS signal strength. Figs. $3 a$ and $b$ show the simulation results for $L_{T}=16$ and $L_{T}=32$, respectively. In both figures, $1 / 4 \leq \alpha \leq 1 / 2$ results in a similar total number of multiplications, but $\alpha=1 / 8$ results in the largest total number of multiplications, which is because the number of iterations $N_{i}$ is increased for smaller $\alpha$. Overall, the computational cost is the lowest or near lowest when $\alpha=1 / 3$, so $L \approx L_{T} / 3$ is a choice leading to a computationally efficient application of the iterative Cadzow denoising algorithm. Therefore, in the following, we use $L \approx L_{T} / 3$ for the proposed technique.

\section{PERFORMANCE ANALYSIS AND NUMERICAL SIMULATIONS}

In this section, we compare the performance of the proposed technique and other techniques including the conventional technique (Tsui 2005).

Exploiting the analysis on the uncertainty of the estimate with the FRI technique for a single Dirac delta function, the variance of the unbiased frequency estimate $\hat{\delta} f$ Eq. (22) is found in Blu et al. (2008) and is lower bounded by the Cramer-Rao lower bound (CRLB) as

$$
\sigma_{\hat{\delta} f}^{2} \geq \frac{3 T_{p}^{-2}}{\pi^{2} L_{T}^{2} P S N R},
$$

where $T_{P}^{-1}=T_{1}^{-1}$ is the period of the frequency domain spectrum, and PSNR represents the peak signal-to-noise ratio such that

$$
\operatorname{PSNR}=\frac{L_{T}\left|E\left[R_{1}(0)^{l}\right]\right|^{2}}{\sigma_{n}^{2}} .
$$

Fig. 4a shows the standard deviation of frequency estimation errors of the proposed technique and other techniques with respect to the carrier to noise density ratio $\left(\mathrm{C} / N_{0}\right)$. The performance of the proposed technique is almost the same to the theoretical bound CRLB when $\mathrm{C} / N_{0}$ is not too weak. Note that when the FFT-based frequency estimation technique Eq. (14) is used, the frequency resolution governs the estimation accuracy so that the 64 -point FFT (i.e., $L_{T}=64$ ) has twice the constant resolution error of the 128 -point FFT as shown in Fig. 4a, where ' $N_{F}$-pt FFT ( $N_{F}$ ms $)^{\prime}$ in the legend denote the $N_{F}$-point FFT from $N_{F}$ ms of data. Similarly, the performance of the serial Doppler frequency search scheme with coherent correlation length $T_{c o}=N_{c o} T_{1} \mathrm{~ms}$ is lower bounded by the Doppler frequency search step size, where $N_{c o}=20$. In the serial Doppler frequency search simulations, we chose the search step size of $10^{3} / 64 \mathrm{~Hz}$ to compare with the 64 -point FFT-based technique. When the curve fitting technique (Zeng \& Li 2010) is applied to the serial Doppler frequency search result obtained with search step size of $1 / 20 \mathrm{~Hz}$, the performance is improved and shows that it has the same slope to the CRLB in the moderate and high $C=N_{0}$ region. Fig. 4a shows the performance of the conventional technique (Tsui 2005) also; the conventional frequency discriminator uses two consecutive ACF outputs, with coherent integration length $T_{1}=1 \mathrm{~ms}$, to compute

$$
\delta f_{\text {conv }}=\frac{1}{2(N-1) \pi T_{1}} \sum_{m=0}^{N-2}\left[I_{m} Q_{m+1}-I_{m+1} Q_{m}\right],
$$

where $I_{m}$ and $Q_{m}$ represent the in-phase and quadraturephase component of the ACF output at $t=m T_{1}$, and the moving average length of $N-1$ is assumed. Note that the performance of the conventional technique is the worst among the techniques shown in Fig. 4 for $C=N_{0}$ not high enough, which is due to the short coherent integration length $T_{1}$ for each ACF output, and that we can expect 3 $\mathrm{dB}$ more performance gain when the coherent integration for each ACF output is doubled. Note also that the ideal frequency estimate in Eq. (28) does not include any measurement error due to the approximation function. After some algebraic manipulations, the frequency error variance can be found as 


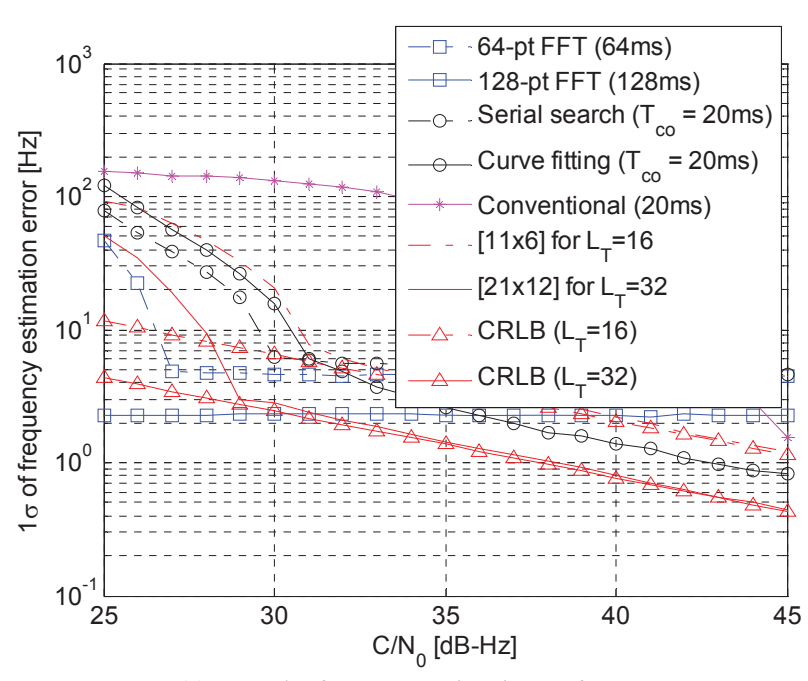

(a) Doppler frequency estimation performance

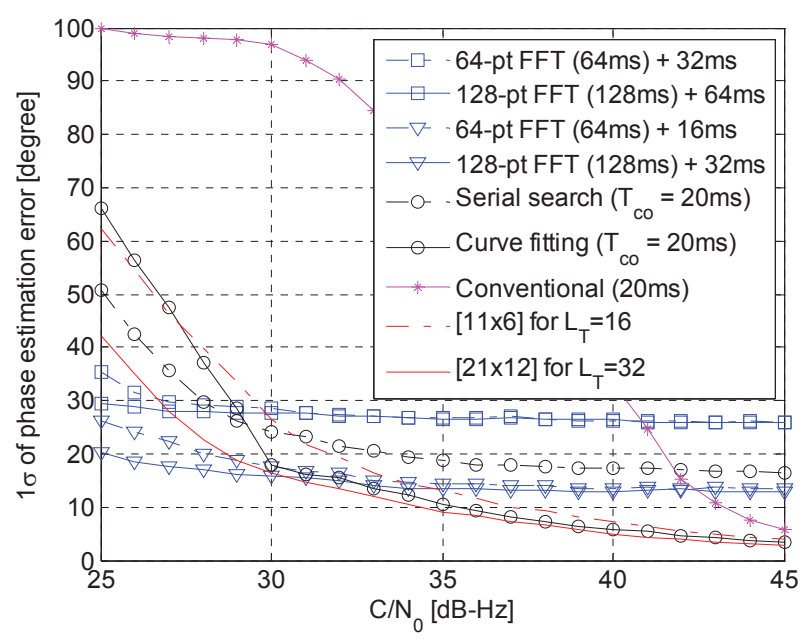

(b) Phase estimation performance

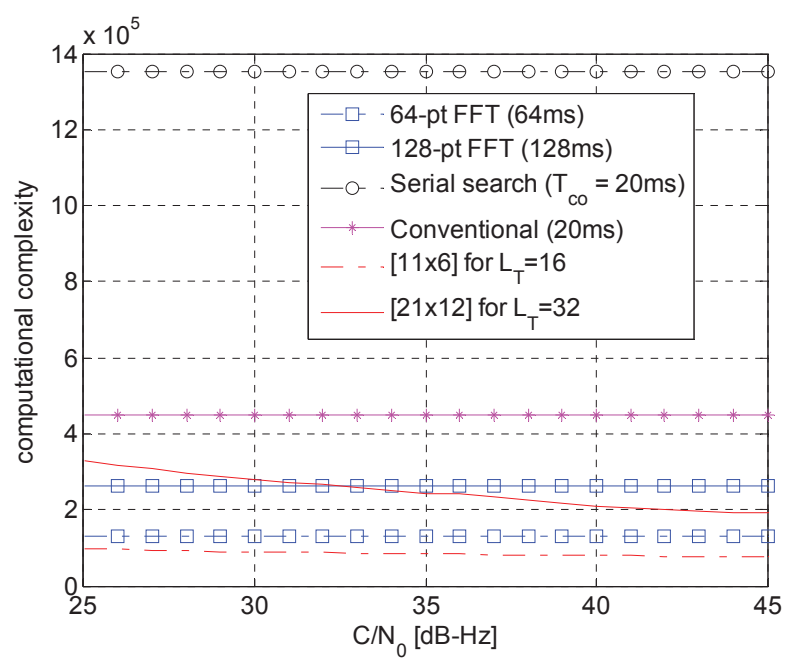

(c) Computational complexity

Fig. 4. Performance comparison.

$$
\operatorname{var}\left\{\delta f_{\text {conv }}\right\}=\frac{1}{4(N-1) \pi^{2} T_{1}^{2} C / N_{0}}\left(1+\frac{1}{2 T_{1} C / N_{0}}\right)
$$

Notice that when $C / N_{0}=45 \mathrm{~dB}-\mathrm{Hz}$ the frequency errors of the proposed technique for $L_{T}=16, L_{T}=32$ and the curve fitting technique are about $1 \mathrm{~Hz}, 0.3 \mathrm{~Hz}$, and $0.8 \mathrm{~Hz}$, respectively, and the errors increase much slowly than in the conventional technique as the $C / N_{0}$ decreases. The resulting phase estimation performance of the techniques compared in Fig. 4a shows some similarity as shown in Fig. 4b. Note that the phase estimation is performed with a shorter data length than the data length used for frequency estimation. For example, when the frequency is estimated with $N_{F}$-point FFT $\left(N_{F}\right)$ ms, the maximum frequency error is $10^{3} / 2 N_{F} \mathrm{~Hz}$ with which the phase error can increase to $\pi[\mathrm{rad}]$. Since a phase estimation with an arc-tangent function may result in a large arithmetic error in the presence of noise, we limit the maximum possible phase estimation error by taking a shorter data length than that for the frequency estimation. In Fig. 4b, a legend, for example, '64-pt FFT (64 ms)+32 ms' denotes the phase estimation with $32 \mathrm{~ms}$ of data when frequency estimation is performed with $L_{T}=64$, and it can be found that a shorter data length for phase estimation results in a smaller phase estimation error. Notice that the performance of phase estimation with the proposed technique and with the curve fitting technique is much better than with other techniques for moderate and strong signals.

Fig. 4c shows the computational complexity (i.e., the number of complex multiplications) of the frequency estimation techniques, such as $N_{F}$-point FFT-based technique $\left(N_{F}=64\right)$, serial Doppler frequency search technique with $T_{c o}=20 \mathrm{~ms}$ and search step size $10^{3} / N_{F}$ $\mathrm{Hz}$, conventional technique Eq. (28) with $N=20$, and the proposed technique with $L_{T}=16 \mathrm{~ms}$ and $32 \mathrm{~ms}$, discussed in this section. When the sampling rate $f_{s}=2 R_{c}=2 \times 1.023$ $\mathrm{MHz}$, the computational costs for the frequency estimation techniques can be expressed as

$$
\begin{aligned}
& M_{F F T}=N_{F}\left(f_{s} T_{1}+\log _{2} N_{F}\right) \\
& M_{S S}=f_{s} T_{c o} N_{D F} \\
& M_{c o n v}=N f_{s} T_{1} \log _{2}\left(f_{s} T_{1}\right) \\
& M_{F R I}=f_{s} T_{1} L_{T}+M_{C D},
\end{aligned}
$$

where $M_{F F T}, M_{S S}, M_{c o n v}$, and $M_{F R I}$ represent the computational complexity of the $N_{F}$-point FFT based technique, the serial search technique with coherent integration length $T_{c o}$ testing $N_{D F}=N_{F} / 2=32$ Doppler frequency hypotheses between the Doppler frequency hypotheses tested with a smaller coherent integration length $T_{1}$ (i.e., 500 
$\mathrm{Hz}$ ), the conventional technique Eq. (28) with $N=20$, and the proposed technique, respectively. Notice that computational complexity for computing the arc-tangent function is not counted in the expressions in Eq. (30), and that, for high $C=N_{0}, M_{F F T}=64 \times\left(2046+\log _{2} 64\right)=131328$, $M_{S S}=20 \times 2046 \times 32=1350360, M_{\text {conv }}=20 \times 2046 \times \log _{2} 2046=450062$, $M_{F R I}=2046 \times 16+5 \times\left[4 \times 11^{2}+22 \times 6^{2}\right] \times 6+\left[4 \times 15^{2}+22 \times 2^{2}\right] \times 2=72992$ for $L_{T}=16$, where $N_{i}=5$, and $M_{F R I}=2046 \times 32+2 \times\left[4 \times 21^{2}+22 \times 12^{2}\right]$ $\times 12+\left[4 \times 31^{2}+22 \times 2^{2}\right] \times 2=191704$ for $L_{T}=32$, where $N_{i}=2$, which match the simulation results for $C / N_{0}=45 \mathrm{~dB}-\mathrm{Hz}$ shown in Fig. 4c.

Obviously, the total complex multiplications of the curve fitting technique are larger than the serial search technique due to the additional curve fitting process. Furthermore, the three figures in Fig. 4 shows that the proposed technique can produce much more accurate Doppler frequency and phase estimates than other techniques and that the computational complexity of the proposed technique is only around $2 \times 10^{5}$ multiplications for $L_{T}$ ms of data, while other techniques have multiple times of estimation error and computational cost in the GPS pull-in state.

\section{CONCLUSIONS}

It has been found that a reverse directional finite rate of innovation technique can be applied to GPS pull-in search and improves the accuracy and computational cost of the pull-in search in comparison to the conventional techniques. Analytical expressions are derived to obtain the theoretical estimates of performance improvement and computational complexity with the proposed technique. The simulations have demonstrated that the proposed technique has a performance improvement when SNR is moderate or high, and that the improvement becomes significant as the resolution of frequency estimation of the proposed technique increases linearly as SNR increases, which is not possible with the conventional FFT-based frequency estimation techniques. In addition to the improvement of the frequency estimation, the proposed technique contributes to the accuracy in the phase estimation, and, therefore, improves the phase tracking performance of a receiver

\section{ACKNOWLEDGMENTS}

This work is supported by the Electronic Warfare Research Center (EWRC) grant funded by the Agency for Defense Development (ADD).

\section{REFERENCES}

Blu, T., Dragotti, P.-L., Vetterli, M., Marziliano, P., \& Coulot, L. 2008, Sparse Sampling of Signal Innovations: Theory, Algorithms and Performance Bounds, IEEE Signal Process Mag., 25, 31-40, http://dx.doi.org/10.1109/ MSP.2007.914998

Borre, K., Akos, D., Bertelsen, N., Rinder, P., \& Jensen, S. 2007, A Software-Defined GPS and Galileo Receiver: A Single-Frequency Approach (Boston, MA: Birkhäuser)

Chan, T. F. 1982, An Improved Algorithm for Computing the Singular Value Decomposition, ACM Trans. Math. Softw., 8, 72-83, http://dx.doi.org/10.1145/355984.355990

Goiser, M. J. \& Berger, G. L. 1996, Synchronizing a digital GPS receiver, MELCON '96, Proc. of the 8th Mediterranean Electrotechnical Conference, May 1996, 1043-1046. http://dx.doi.org/10.1109/MELCON.1996.551387

Golub, G. H. \& van Loan, C. F. 1996, Matrix Computations, 3rd ed. (Baltimore: The Johns Hopkins Univ. Press)

Hayes, M. H. 1996, Statistical Digital Signal Processing and Modeling. (New York: John Wiley \& Sons)

Irsigler, M. \& Eissfeller, B. 2003, Comparison of Multipath Mitigation Techniques with Consideration of Future Signal Structures, in Proc. of ION GPS/GNSS 2003, Portland, OR., Sep 2003

Kelley, C., Cheng, J., \& Barnes, J. 2002, Open source software for learning about GPS, in Proc. of ION GPS/GNSS 2002, Portland, OR., Sep 2002

Kim, B., Muchkaev, A., \& Kong, S.-H. 2011, SAR Image Processing Using Super-Resolution Spectral Estimation with Annihilating Filter, 2011 3rd International AsiaPacific Conference on Synthetic Aperture Radar, APSAR, Seoul, Korea, 1-4 Sep 2011

Kong, S.-H. \& Kim, B. 2013, Two-Dimensional Compressed Correlator for Fast PN Code Acquisition, IEEE Trans. Wirel. Commun., 12, 5859-5867, http://dx.doi. org/10.1109/TWC.2013.092313.130407

Kransner, N. F. 1998, GPS receiver and method for processing GPS signals, US Patent, PN 5,781,156

Parkinson, B., Spilker, J., Axelrad, P., \& Enge, P. 1996, Global Positioning System: Theory and Applications (Washington, DC: American Institute of Aeronautics and Astronautics)

Petovello, M., O'Driscoll, C., \& Lachapelle, G. 2008, Weak Signal Carrier Tracking Using Extended Coherent Integration with an Ultra-Tight GNSS/IMU Receiver, in Proc. of ENC 2008, Toulouse, Apr 2008

Pisarenko, V. F. 1973, The Retrieval of Harmonics from a Covariance Function, Geophys. J. R. Asfr. Soc., 347-366, Sep 1973 
Psiaki, M. L. \& Jung, H. 2002, Extended Kalman Filter Methods for Tracking Weak GPS Signals, in Proc. of ION GPS/ITM 2002, Portland, OR, 2539-2553 Sep 2002

Sagiraju, P. K., Akopian, D., \& Valio, H. 2006, Fine Frequency Estimation in Weak Signals for GPS Receivers, in Proc. of ION GPS/NTM 2006, Monterey, CA, 2524-2533 Jan 2006

Tsui, J. B. Y. 2005, Fundamentals of Global Positioning Receivers: A Software Approach, 2nd Ed. (New York: John Willey \& Sons)

Tufts, D. W. \& Kumaresan, R. 1982, Estimation of frequencies of multiple sinusoids: Making linear prediction perform like maximum likelihood, Proc. IEEE, 975-989 Sep 1982, http://dx.doi.org/10.1109/PROC.1982.12428

van Diggelen, F. 2009, A-GPS: Assisted GPS, GNSS, and SBAS. (Norwood, MA: Artech House)

Vetterli, M., Marziliano, P., \& Blu, T. 2002, Sampling Signals with Finite Rate of Innovation, IEEE Trans. Signal Process, 50, 1417-1428, http://dx.doi.org/10.1109/ TSP.2002.1003065

Zeng, D. \& Li, J. 2010, GPS Signal Fine Acquisition Algorithm, Information Science and Engineering (ICISE), 2010 2nd International Conference on IEEE, 3729-3732 Dec 2010, http://dx.doi.org/10.1109/ICISE.2010.5691791

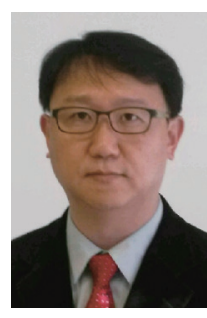

Seung-Hyun Kong received a B.S.E.E. from the Sogang University, Korea, in 1992, an M.S.E.E. from the Polytechnic University, New York, in 1994, and a Ph.D. degree in Aeronautics and Astronautics from Stanford University, CA, in 2006. From 1997 to 2004, he worked with Samsung Electronics Inc. and Nexpilot Inc., both in Korea, where his research focus was on 2G CDMA and 3G UMTS PHY and mobile positioning technologies. In 2006, he was involved with the development of hybrid positioning technology using wireless location signature and Assisted GNSS at Polaris Wireless, Inc. and from 2007 to 2009, he was a researcher at Qualcomm Research Center, San Diego, CA, where his R\&D focus was on indoor location technologies and advanced GNSS technologies. Since 2010, he has been an Assistant Professor at the CCS Graduate School for Green Transportation in the Korea Advanced Institute of Science and Technology (KAIST). His research interests include super-resolution signal processing, detection and estimation for navigation systems, and Assisted GNSS in wireless communication systems.

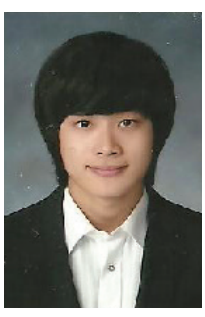

Kyungwoo Yoo received a B.S.E.E from Inha University, Korea, in 2012, and a M.S. degree from the CCS Graduate School for Green Transportation in the Korea Advanced Institute of Science and Technology (KAIST), Korea, in 2014. He is currently pursuing a Ph.D. degree with the same institution. His research interests include anti-jamming technique and signal processing for GNSS and navigation system. 